Methods 36 years old, referred to our emergency department at the beginning of January 2020 as suspected ectopic pregnancy on ultrasound and plateauing BHCG of $1012 \mathrm{mIU} / \mathrm{ml}$. had 5 weeks amenorrhea, no vaginal bleeding, her Last delivery was vaginally in august 2019. Was given methotrexate 2 doses, with no response, a diagnostic laparoscopy, and examination under anesthesia done, which found no ectopic pregnancy and a $5 \times 3 \mathrm{~cm}$ vaginal mass noticed. Biopsy taken showed choriocarcinoma. Started on combination chemotherapy, responded well her BHCG became $<1$, still under follow up.

Results Vaginal metastasis of trophoblastic tumor may occur even after vaginal delivery. This case was erroneously diagnosed as ectopic pregnancy and diagnosed during surgical intervention. Chemotherapy is the treatment of choice with a favorable prognosis. Regarding prognostic scoring, vaginal metastasis should be considered as a poor prognostic factor. Different studies in this context thus directly recommended combination chemotherapy as their first choice.

Conclusions While dealing with a case of vaginal mass with a history of antecedent pregnancy and rising BHCG, possibility of metastatic choriocarcinoma should be considered and investigate accordingly. Prompt diagnosis and early treatment with combination chemotherapy may thus save many lives.

\section{EPV283/\#72 DEVELOPMENT OF A GESTATIONAL TROPHOBLASTIC NEOPLASIA REGISTRY AND PROTOCOL IN AN OBGYN RESIDENCY IN RWANDA}

${ }^{1} \mathrm{~L}$ Bazzett-Matabele*, ${ }^{2} \mathrm{~B}$ Dushimiyimana, ${ }^{3} \mathrm{G} \quad$ Uwitonze, ${ }^{4} \mathrm{~T}$ Sebazungu, ${ }^{4} \mathrm{D}$ Ntasumbumuyange, ${ }^{4} \mathrm{~S}$ Rulisa, ${ }^{5} \mathrm{M}$ Small, ${ }^{6} \mathrm{U}$ Magriples, ${ }^{7} \mathrm{R}$ Ghebre. ${ }^{1}$ University of Botswana, Obgyn, Gaborone, Botswana; ${ }^{2}$ Ruhengeri District Hospital, Obgyn, Ruhengeri, Rwanda; ${ }^{3}$ Kibagabaga District Hospital, Obgyn, Kigali, Rwanda; ${ }^{4}$ University of Rwanda, Obgyn, Kigali, Rwanda; ${ }^{5}$ Duke University, Obgyn, Durham, USA; ${ }^{6}$ Yale University, Obgyn, New Haven, USA; ${ }^{7}$ University of Minnesota, Obgyn, Minneapolis, USA

\subsection{6/ijgc-2021-IGCS.354}

Objectives Gestational Trophoblastic Neoplasia (GTN) cure rates reach $>90 \%$ in settings where early diagnosis and management strategies exist. GTN is more common in African countries, where many factors effecting outcomes are not readily available. To address the high prevalence of invasive molar pregnancies in Rwanda we developed training in sonographic recognition, clinical diagnosis and management of GTN in the largest teaching hospital in Kigali, Rwanda.

Methods We evaluated our approach to GTN management in the largest tertiary care teaching hospital in Rwanda.

Results A patient registry of GTN patients was created with gynecologic oncology specialists. From October 2015 to June 2019 we identified 108 patients with GTN, 80 of which were diagnosed with invasive mole. Residents at all levels received training in ultrasound recognition of invasive versus noninvasive mole characteristics, GTN staging and scoring, methotrexate dosing and toxicity, B-hCG monitoring and identification of high risk or resistant disease. Residents were also trained in the appropriate use of hysterectomy in the management of Gestational Trophoblastic Disease.

Conclusions Recently trained $\mathrm{OB} / \mathrm{GYN}$ residents practicing at hospitals countrywide are now able to identify and refer appropriate patients to the GTN center at the university teaching hospital in Kigali, Rwanda. Based on these results we feel that appropriate GTN diagnosis and management can be taught in a low resource setting, even outside of the university teaching hospital, to improve patient outcomes despite limited resources.

\section{EPV284/\#238 THE EARLY DETECTION OF VULVAR CANCER THROUGH SELF-EXAMINATION (EDUCATE) STUDY: WHAT WOMEN AND CLINICIANS THINK}

${ }^{1}$ VN Sivalingam*, ${ }^{1} \mathrm{~K}$ Tamber, ${ }^{2} \mathrm{~J}$ Newsham, ${ }^{2} \mathrm{~S}$ Ogden, ${ }^{3} \mathrm{U}$ Winters, ${ }^{4,5} \mathrm{FM}$ Walter, ${ }^{1} \mathrm{RJ}$ Edmondson, ${ }^{1} \mathrm{EJ}$ Crosbie. ${ }^{1}$ University of Manchester, Division of Cancer Sciences, School of Medical Sciences, Manchester, UK; ${ }^{2}$ Salford Royal NHS Foundation Trust, The Dermatology Centre, Manchester, UK; ${ }^{3}$ Manchester University Hospitals NHS Foundation Trust, Colposcopy and Gynaecological Oncology, Manchester, UK; ${ }^{4}$ University of Cambridge, Department of Public Health and Primary Care, Cambridge, UK; ${ }^{5}$ Queen Mary University of London, Institute of Population Health Sciences, London, UK

\subsection{6/ijgc-2021-IGCS.355}

Objectives Rates of vulvar cancer are increasing globally. Early detection reduces surgical morbidity and prolongs survival. Although population screening has no role, vulvar self-examination may prompt early diagnosis in high-risk women. UK guidance promotes self-examination in women with high-risk vulvar conditions, but there is a lack of evidence about current practice, acceptability and barriers to vulvar selfexamination.

Methods Clinician questionnaires were completed at a UK vulvar conference. Patient questionnaires (incorporating vulvar self-examination and cancer awareness) were distributed through patient networks and clinics.

Results All ninety-eight clinicians agreed that self-examination plays an important role in detecting sinister vulvar changes in high-risk women. $87 \%$ recommended monthly self-examination and $81 \%$ provided one-to-one teaching despite believing that few patients practised self-examination. 455 patients (median age 58 years) with lichen sclerosus(69\%), lichen planus(13\%), vulvar cancer(14\%) and VIN(13\%) participated. Clinic respondents $(n=197)$ were older(median 65 vs 52 years, $\mathrm{p}<0.001)$ and $65 \%$ reported self-examining compared with $86 \%$ of online respondents $(\mathrm{p}<0.001)$. Despite regular selfexamination, $40 \%$ were not confident about recognising vulvar abnormalities. Lack of awareness(38\%), confidence(31\%) and physical difficulties visualising the vulva(32\%) were top barriers to self-examination. Face-to-face specialist teaching was regarded as the best way to learn self-examination but only $9 \%$ of patients reported receiving this. Patients agreed that a magnified, extendable mirror and photographs depicting sinister changes would aid self-examination.

Conclusions Patients and clinicians recognise that vulvar selfexamination is important in early detection of cancer, but a lack of formal teaching impairs confidence in the identification of abnormalities. Visual aids may facilitate self-examination but should be reinforced by education and support.

\section{EPV285/\#322 HUMAN LEUKOCYTE ANTIGEN-G EXPRESSION IN VULVAR SQUAMOUS CELL CARCINOMA}

${ }^{1,2} \mathrm{~N}$ Boujelbene* ${ }^{2,3} \mathrm{I}$ Zemni, ${ }^{2} \mathrm{~W}$ Babay, ${ }^{2} \mathrm{H}$ Ben Yahia, ${ }^{2} \mathrm{~S}$ Dhouioui, ${ }^{1} \mathrm{~K}$ Mrad, ${ }^{2} \mathrm{HI}$ Ouzari, ${ }^{1} \mathrm{~L}$ Charfi, ${ }^{2}$ Z Zidi. 'Salah Azaiez Institute of Cancerology, University of Tunis El Manar, Department of Pathology, Tunis, Tunisia; ${ }^{2}$ University of Tunis El Manar,Sciences Faculty of Tunis, Laboratory of Microorganismes and Active Biomolecules, Tunis, Tunisia; ${ }^{3}$ Salah Azaiez Institute of Cancerology, University of Tunis El Manar, Department of Surgical Oncology, Tunis, Tunisia

10.1136/ijgC-2021-IGCS.356 